\title{
MODELADO Y SIMULACIÓN DE UN SISTEMA DE ENFRIAMIENTO PARA MANTENER LA TEMPERATURA DE TRABAJO DE UN DINAMÓMETRO HIDRÁULICO.
}

\section{MODELING AND SIMULATION OF A COOLING SYSTEM TO MAINTAIN THE WORKING TEMPERATURE OF AN HYDRAULIC DYNAMOMETER}

\author{
Ing. Frank Wiliam Blanco Ojeda*, Ing. Yeyner Alveiro Carrillo Pabón*, \\ Msc. José Ricardo Bermúdez Santaella*, Msc. David Fernando Marcucci Pico**
}

* Universidad Francisco de Paula Santander, programa ingeniería electromecánica, grupo de investigación de desarrollo de procesos industriales (GIDPI).

Avenida gran Colombia No. 12E-96 Barrió Colsag, San José de Cúcuta, Norte de

Santander, Colombia. +573123289641.

E-mail: \{frankwiliamadlfobo, yeyneralveirocp, josericardobs $\} @$ ufps.edu.co.

** Universidad Federal de Uberlândia, Programa ingeniería mecánica, Laboratorio de sistemas térmicos y nanotecnología.

Av João Naves de Ávila, 2121, Uberlândia, Minas Gerais, Brasil.

$+553432394074$

E-mail: dmarcucci@ufu.br

Resumen: Este documento describe el procedimiento para el modelado y simulación de un sistema de enfriamiento para mantener la temperatura de trabajo de un dinamómetro hidráulico, este dispositivo es utilizado para la caracterización de motores de combustión interna en términos de velocidad, torque y potencia. Durante este proceso se aumenta la temperatura en el fluido de trabajo del dinamómetro hidráulico debido a la carga ejercida al motor de combustión de interna, dicho aumento de temperatura afecta el correcto funcionamiento del dinamómetro, por lo que se hace necesario emplear un sistema de enfriamiento, el cual es modelado e implementado en la herramienta Matlab-simulink ${ }^{\circledR}$ a través de las ecuaciones que rigen su comportamiento, por último se realizó la validación del modelo matemático a través de la comparación de datos experimentales, observando que el error fue de $4.6 \%$, obteniendo de esta forma una herramienta validada para el sistema de enfriamiento del dinamómetro hidráulico.

Palabras clave: Calor, Dinamómetro hidráulico, Sistema de Enfriamiento, Radiador, Temperatura.

\begin{abstract}
This document describes the procedure for the modeling and simulation of a cooling system to maintain the working temperature of a hydraulic dynamometer, this device is used for the characterization of internal combustion engines in terms of speed, torque and power. During this process, the temperature in the working fluid of the hydraulic dynamometer is increased due to the load exerted on the internal combustion engine, this increase in temperature affects the correct operation of the dynamometer, so it is necessary to use a cooling system, which is modeled and implemented in the Matlabsimulink ${ }^{\circledR}$ tool through the equations that govern its behavior, finally the validation of the mathematical model was made through the comparison of experimental data, observing that the error was $4.6 \%$, obtaining in this way a validated tool for the cooling system of the hydraulic dynamometer.
\end{abstract}

Keywords: Heat, Hydraulic Dynamometer, Cooling System, Radiator, Temperature. 


\section{INTRODUCCIÓN}

Los motores de combustión interna son los elementos de propulsión más utilizados en los automóviles, con el aumento en la competencia en los diversos diseños de motores los fabricantes se ven obligados en investigar nuevos proyectos que permitan obtener dispositivos con una mayor eficiencia. Para el estudio de los motores de combustión interna, se utilizan los dinamómetros, en este caso de estudio se utilizó un dinamómetro hidráulico. El funcionamiento de este equipo consiste en generar una carga en los motores de combustión interna, y así obtener las variables más relevantes como el torque, potencia y velocidad. (Ariza F y Vanegas R, 2013) (RÁ López, MG Angarita. (2017).

El dinamómetro hidráulico con el que cuenta el laboratorio fue construido en Bogotá por la empresa Dising Industrial con los planos que fueron realizados en el trabajo de grado de (Estupiñan y Franco, 2014). Cuando se realizan pruebas con el dinamómetro hidráulico se debe asegurar que el dispositivo se encuentre trabajando en régimen estable (es decir que la recamara del equipo se encuentre totalmente llena del fluido de trabajo) ya que de lo contrario se presentara el fenómeno de auto vaciado en el dinamómetro hidráulico, esto quiere decir que el instrumento se encuentra operando en régimen transitorio, este efecto sucede debido a que el equipo se comporta como una bomba centrifuga, haciendo que el flujo de salida sea mayor que el de entrada. (Hodson, 1991) (KCP López, BM Delgado. 2017).

Cuando se estipula la carga en el motor de combustión interna y una vez asegurado que el dinamómetro trabaja en la zona de la estabilidad, empiezan a ocurrir tres efectos internos, como lo son el efecto de la cantidad de movimiento, el efecto de la viscosidad y el efecto de la turbulencia. (Canizales y Zapata, 2012). Estos efectos ocasionan un aumento en la temperatura en el fluido de trabajo del dinamómetro. Se investigó en textos especializados y se encontró en el artículo de (Narayan, 1968) que se recomienda que a la entrada y la salida del dinamómetro hidráulico la variación de temperatura deba estar entre $50{ }^{\circ} \mathrm{C}$ y $70{ }^{\circ} \mathrm{C}$. En este sentido, se concluye que el equipo debe trabajar a temperaturas inferiores a $70{ }^{\circ} \mathrm{C}$ o en su defecto se deba mantener la temperatura a un valor específico, con el fin de evitar que se generen daños por cavitación, debido al cambio en las propiedades del fluido de trabajo (agua).
Se realizaron pruebas en régimen estacionario en el banco de pruebas de motores, que contiene el dinamómetro hidráulico en análisis, en donde se evidencia que la temperatura del fluido de trabajo puede alcanzar valores por encima de los $70{ }^{\circ} \mathrm{C}$ como se muestra en la Fig. 1. Por lo tanto se justifica la necesidad emplear un sistema de enfriamiento que permita una apropiada remoción de calor y en consecuencia que la temperatura del fluido de trabajo (agua) sea más baja, para garantizar que el dinamómetro hidráulico y bomba centrifuga funcionen en óptimas condiciones, Ya que de lo contrario se pueden causar fallas por desgaste y erosión en los sellos mecánicos, o'rings, $\mathrm{y}$ alabes de este dispositivo.

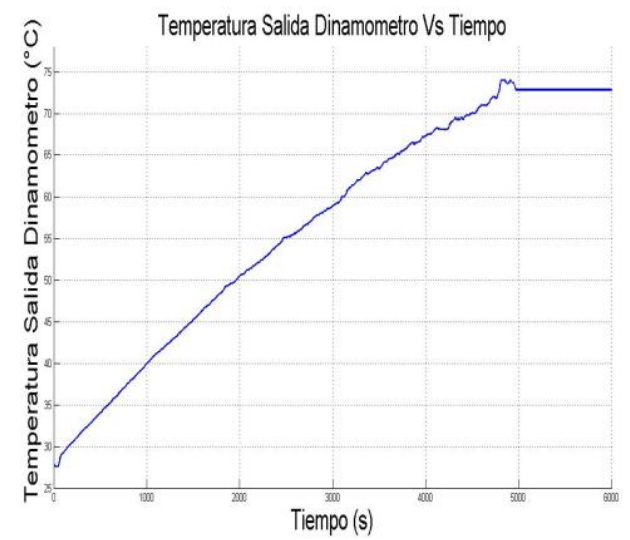

Fig. 1 Comportamiento de la temperatura del fluido de trabajo en el dinamómetro hidráulico. Fuente autores

En solución a dicha problemática se presenta el estudio de un sistema de enfriamiento para un dinamómetro hidráulico, en el cual se analizó un radiador comercial que tiene como fin reducir la temperatura del fluido que circula a la entrada del dinamómetro, para cumplir con tal objetivo se obtuvieron las ecuaciones que expresan el comportamiento del sistema de enfriamiento, se aplicaron los conceptos de la termodinámica, relacionados a la conservación de masa y la primera ley de la termodinámica. Seguidamente se implementó el modelo matemático del sistema de enfriamiento en el software Matlab-simulink ${ }^{\circledR}$, este programa es fundamental para la simulación de sistemas dinámicos ya que ofrece la capacidad de conocer el comportamiento de un proceso por medio de modelos matemáticos, para en efecto reducir los costos generados en pruebas experimentales y posteriormente, su utilización en el área de la investigación. (Mejia et al., 2003) 


\section{SISTEMA DE ENFRIAMIENTO PARA EL DINAMÓMETRO HIDRÁULICO}

El radiador utilizado es un intercambiador de calor del tipo flujo cruzado agua-aire con ambos fluidos sin mezclar. El objetivo principal del radiador es retirar el calor del fluido caliente, agua que circula por medio de tubos con aletas en contacto con directo con un fluido externo, aire a temperatura ambiente. El flujo de aire que circula por el lado externo del radiador es generado por un ventilador, accionado por un motor de corriente continua de 12 V.

En la Fig. 2 se presenta el banco de pruebas, el cual está compuesto por: (1) motor de combustión interna, (2) dinamómetro hidráulico, (3) sensor encoder, (4) celda de carga, (5) reservatorio y (6) el radiador. El sensor encoder es utilizado para medir las revoluciones del motor y la celda de carga es utilizada para medir la fuerza (torque del motor).

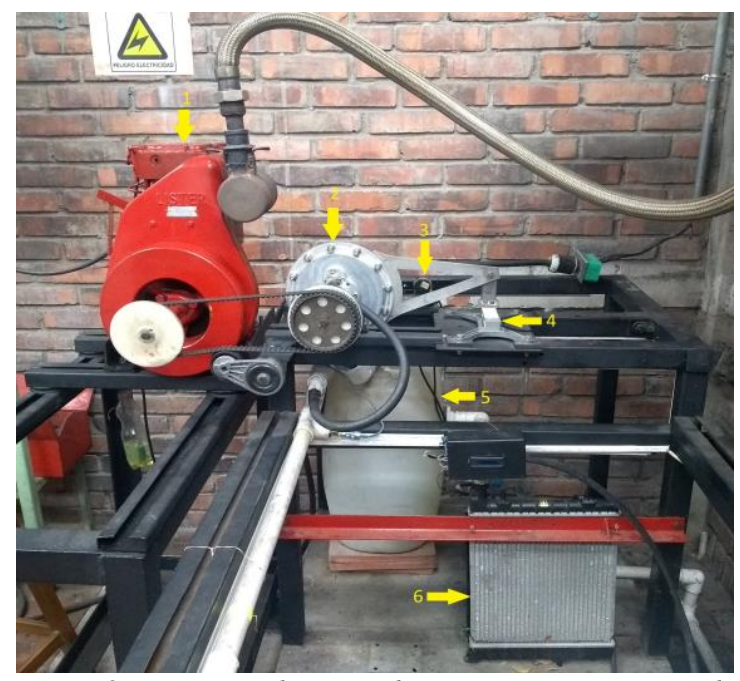

Fig. 2 Banco de pruebas para motores de combustión interna. Fuente autores.

\section{MODELADO DEL SISTEMA DE ENFRIAMIENTO}

Para determinar el modelo simplificado del sistema de enfriamiento se realizó un balance de energía en el intercambiador de calor, donde el fluido que circula internamente es el agua y externamente intercambia calor con un flujo de aire. Para la obtención de algunos de los parámetros relevantes del radiador, tales como: flujo másico del aire, temperatura del aire a la entrada y salida del radiador, fueron realizados algunos ensayos experimentales utilizando un anemómetro y dos sensores de temperatura ubicados a la entrada y la salida del radiador, respectivamente.

\subsection{Balance de energía del radiador por el lado} del aire

Para calcular la tasa de incremento de energía del aire que circula a través del radiador, se debe cuantificar el valor de $Q_{\text {aire }}$ que expresa el calor que disipa el radiador cuando se encuentra el ventilador operando a máxima velocidad, dicha energía puede calcularse utilizando la primera ley de la termodinámica aplicada únicamente al lado del aire, como se expresa en la Ecuación (1):

$$
\dot{Q}_{\text {aire }}=\dot{m}_{\text {aire }} c_{\text {paire }}\left(T_{\text {sair }}-T_{\text {eair }}\right)
$$

Donde $\stackrel{\square}{m_{\text {aire }}}$ es el flujo másico de aire externo producido por el ventilador, $C_{\text {paire }}$ es el calor específico a presión constante del aire, $T_{\text {sair }}$ y $T_{\text {eair }}$ respectivamente, representan la temperatura del aire a la salida y entrada del radiador.

Para la obtención del calor del aire se debe obtener la temperatura media del aire que a pasa a través del radiador (Romero y Carranza, 2007), dicha temperatura se calcula por medio de la Ecuación (2):

$$
T_{\text {aire }}=\frac{T_{s}+\frac{T_{e n t r}+T_{s a l}}{2}}{2}
$$

Donde $T_{s}$ es la temperatura en la aleta del radiador la cual fue medida con un multímetro digital con termocupla marca M890g, $T_{\text {entr }}$ es la temperatura ambiente del radiador, $T_{\text {sal }}$ es la temperatura a la salida del ventilador, estas dos últimas fueron tomadas por el anemómetro.

Una vez determinada la temperatura media del aire se procede a calcular por medio del software EES, el valor de $C_{\text {paire }}$.

En última instancia Para calcular el flujo másico de aire $\left(\stackrel{\square}{Q}_{\text {aire }}\right)$ que atraviesa el radiador fue utilizado un balance de materia, como es expresado en la Ecuación (3):

$$
\dot{m}_{\text {aire }}=\rho_{\text {aire }} V_{\text {aire }} A_{\text {ventilador }}
$$

Donde $\rho_{\text {aire }}$ representa la densidad del aire a la temperatura y presión atmosféricas, $V_{\text {aire }}$ representa la velocidad del aire a la salida del radiador, $\mathrm{La}$ 
velocidad del aire fue determinada de forma experimental utilizando un anemómetro digital, del fabricante Minipa, modelo MDA-11 y con precisión de $3 \%$ de la faja de medición. Para determinar la velocidad del aire fueron realizadas 30 mediciones de velocidad de forma aleatoria, alternando los puntos de medición en ventilador, como indicado en la Fig. 3, con el fin de estimar la velocidad promedio del aire que fluye por el exterior del radiador.

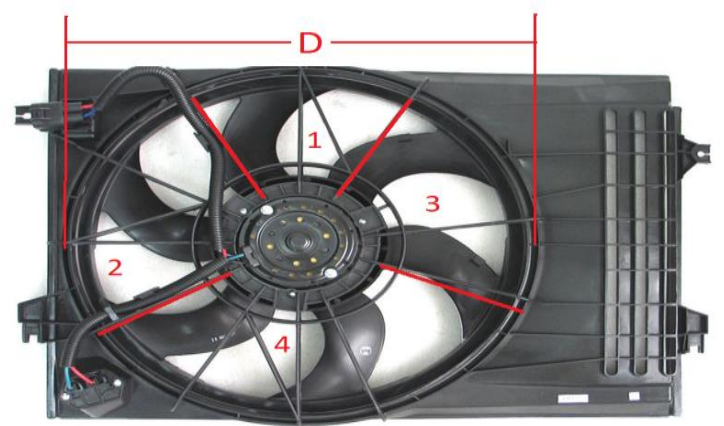

Fig. 3 Ventilador que está conectado al radiador. Fuente autores.

El área fue determinada por el diámetro de la descarga del ventilador, como lo indica la fig. 3. Una vez conocido el diámetro se calcula el área por donde circula el aire a través de la Ecuación (4).

$$
A_{\text {ventilador }}=\frac{\pi}{4}(D)^{2}
$$

\subsection{Balance de energía del agua que circula a través del radiador}

Al hacer un balance de energía en el radiador, teniendo en cuenta que la energía cedida por el agua $\left(\stackrel{\square}{Q}_{\text {agua }}\right)$ debe ser aproximadamente igual a la energía absorbida por el aire $\left(\stackrel{\square}{Q}_{\text {aire }}\right)$ que atraviesa el radiador, se asume que $\stackrel{\square}{Q}_{\text {aire }}=\stackrel{\square}{Q}_{\text {aви }}$ (Verdesoto, 2006). La tasa de transferencia de calor cedida por el lado del agua puede ser calculada por medio de la Ecuación (5).

$$
\stackrel{\square}{\text { agua }}=q \rho_{\text {agua }} C_{\text {pagua }}\left(T_{\text {salagu }}-T_{\text {entragu }}\right)
$$

Donde $\underset{q}{q}$ es el flujo volumétrico de agua que circula por el dinamómetro hidráulico, $\rho_{\text {agи }}$ es la densidad media del agua, $C_{\text {pagua }}$ es el calor específico a presión constante del agua, $T_{\text {salagu }} \mathrm{y}$ $T_{\text {entragu }}$ representan, respectivamente, la temperatura de salida y de entrada del radiador.

\subsection{Obtención de la ecuación dinámica del radiador}

Para hallar la ecuación dinámica del radiador se abordó el siguiente volumen de control, tal y como se muestra en la Fig. 4

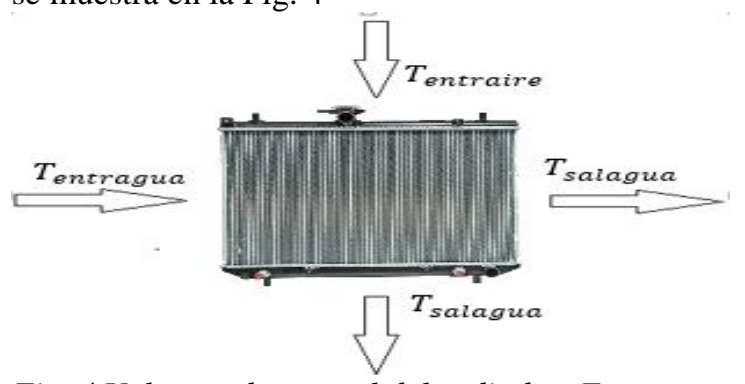

Fig. 4 Volumen de control del radiador. Fuente autores.

Aplicando balances de energía, balance de masa, para el sistema de enfriamiento (Çengel y Boles, 2012). Se planteó la ecuación general de conservación de la energía y se obtuvo la Ecuación.

(6) $\frac{d U(t)}{d t}=E_{\text {entr }}(t)-E_{\text {sal }}(t)$

Donde $\frac{d U(t)}{d t}$ representa la derivada con respecto al tiempo de la energía en un proceso termodinámico, $E_{\text {entr }}(t)$ y $E_{\text {sal }}(t)$ representan la energía de entrada y salida del sistema.

Reemplazando los respectivos valores y agrupando términos se obtiene la Ecuación (7):

$\frac{d T_{\text {salagu }}}{d t}=\frac{m_{\text {aire }} C_{\text {pair }}\left(T_{\text {eair }}-T_{\text {sair }}\right)+m_{\text {agua }} C_{\text {pagua }}\left(T_{\text {entrag }}-T_{\text {salagu }}\right)}{\rho V C_{\text {vagu }}}$ (7)

Para solventar la Ecuación (7) se tuvo en cuenta que el volumen del agua total del sistema del sistema de refrigeración, tales como: $V_{\text {resevatorio }}$ que representa el volumen de agua del reservatorio, $V_{\text {radiador }}$ que representa el volumen de agua del radiador y $V_{\text {tuberias }}$ que representa el volumen del agua de las tuberías, dando como resultado la Ecuación (8)

$$
V=V_{\text {reservatorio }}+V_{\text {radiador }}+V_{\text {tuberias }}
$$

Para el cálculo de la velocidad del aire se implementó la siguiente Ecuación (9), que expresa el comportamiento del ventilador con respecto al tiempo.

$$
\frac{d V_{\text {aire }}}{d t}=\frac{1}{\lambda}\left(V_{\text {vent }}-V_{\text {aire }}\right)
$$


Donde $\lambda$ es el tiempo de respuesta en el que ventilador alcanza su velocidad nominal, $V_{\text {vent }}$ es la velocidad del ventilador determinado teóricamente por la linealidad con el voltaje aplicado al ventilador, $V_{\text {aire }}$ es la velocidad del aire determinada experimentalmente por el anemómetro.

\section{IMPLEMENTACIÓN DEL MODELO EN SOFTWARE MATLAB-SIMULINK ${ }^{\circledR}$}

Antes de implementar el modelo matemático del sistema de enfriamiento, primeramente se igualan las Ecuaciones (7) y (9) a cero para determinar las condiciones naturales para el radiador y ventilador respectivamente. Seguidamente, se procede a evaluar el modelo matemático en el software Matlab-Simulink ${ }^{\circledR}$. Para visualizar el comportamiento y estabilidad del sistema a través de la simulación.

En la Fig. 5 se muestra la implementación por diagrama de bloques la Ecuación (9) que representa la velocidad del ventilador.

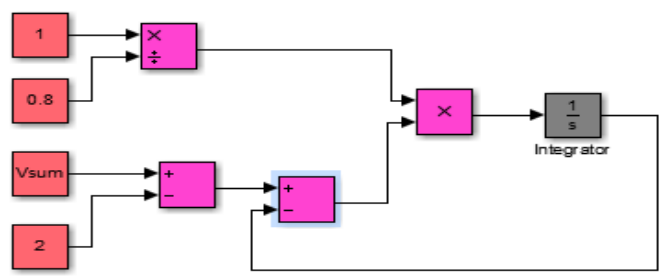

Fig. 5 Representación de la ecuación dinámica del ventilador. Fuente autores

En la Fig. 6 Se muestra el comportamiento dinámico de la velocidad del ventilador que representa la fig. 5, esta respuesta se obtuvo al excitar el sistema con una fuente de voltaje, dando como resultado una estabilidad en la velocidad de $8.4670 \mathrm{~m} / \mathrm{s}$ a una alimentación de $12 \mathrm{v}$ para el ventilador.

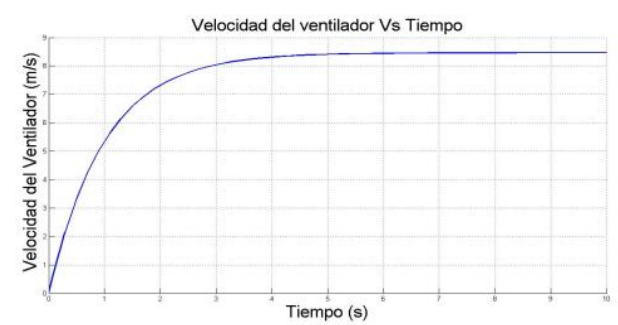

Fig. 6 Respuesta del sistema ante una excitación de voltaje al ventilador del radiador. Fuente autores.
En la Fig. 7 se muestra el diagrama de bloques de la Ecuación (7), este representa el modelo matemático del sistema de enfriamiento. Donde el subsistema de color azul claro representa la velocidad del aire, el subsistema de color azul oscuro representa la energía del fluido frio, el subsistema de color rojo representa la energía del fluido caliente y el subsistema de color gris representa la energía interna a la salida del sistema que se obtuvo al despejar la temperatura de salida.

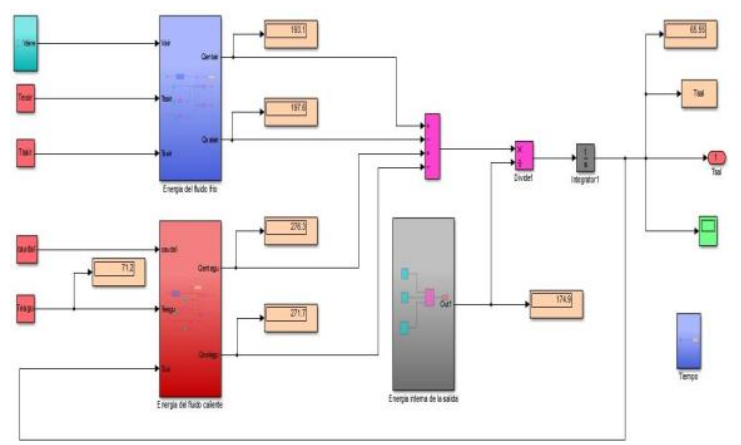

Fig. 7 Representación de la ecuación dinámica del radiador. Fuente autores.

A continuación en la Fig. 8 Se muestra el comportamiento dinámico del sistema de enfriamiento, para obtener esta respuesta se esperó un tiempo determinado en el que el sistema alcanza la estabilidad en la temperatura la cual es de 72.5 ${ }^{\circ} \mathrm{C}$, seguidamente se enciende el ventilador del radiador para observar el comportamiento transitorio en la temperatura del sistema de enfriamiento dando como resultado una temperatura de $65.56{ }^{\circ} \mathrm{C}$.

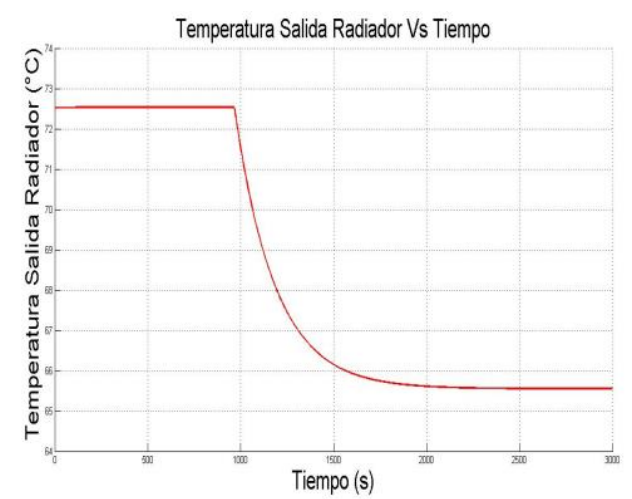

Fig. 8 Respuesta del sistema ante la excitación de velocidad del aire que circula a través del radiador. Fuente autores.

En la Tabla 1 se muestra los parámetros que fueron incluidos al modelo matemático con el fin de 
obtener el comportamiento de la figuras.

Tabla 1 parámetros que fueron incluidos al modelo matemático. Fuente autores.

\begin{tabular}{lcc}
\hline ÍTEM & Valor & $\begin{array}{c}\text { Unidades } \\
\text { SI }\end{array}$ \\
\hline Calor especifico aire & 1.005 & {$[\mathrm{~kJ} / \mathrm{kg} \cdot \mathrm{K}]$} \\
Temperatura de entrada & 25 & {$\left[{ }^{\circ} \mathrm{C}\right]$} \\
Temperatura de salida & 32 & {$\left[{ }^{\circ} \mathrm{C}\right]$} \\
Temperatura de entrada & 72.5 & {$\left[{ }^{\circ} \mathrm{C}\right]$} \\
Densidad del aire & 1.1729 & {$\left[\mathrm{~kg} \cdot \mathrm{m}^{-3}\right]$} \\
Densidad del agua & 976.3 & {$\left[\mathrm{~kg} \cdot \mathrm{m}^{-3}\right]$} \\
Volumen de agua del radiador & 0.000752 & {$\left[\mathrm{~m}^{3}\right]$} \\
Volumen de agua del reservatorio & 0.0524 & {$\left[\mathrm{~m}^{3}\right]$} \\
Volumen de agua por las tuberías & 0.001718 & {$\left[\mathrm{~m}^{3}\right]$} \\
Calor especifico agua & 4.174 & {$[\mathrm{~kJ} / \mathrm{kg} \cdot \mathrm{k}]$} \\
caudal & 15 & {$[\mathrm{LPM}]$} \\
Calor volumen constante & 4.1 & {$\left[\mathrm{kj} / \mathrm{kg}{ }^{\circ} \mathrm{c}\right]$} \\
Velocidad del aire & 8.4670 & {$[\mathrm{~m} / \mathrm{s}]$} \\
Flujo másico de aire & 0.6852 & {$[\mathrm{~kg} / \mathrm{s}]$} \\
Flujo másico de agua & 0.1919 & {$[\mathrm{~kg} / \mathrm{s}]$} \\
\hline
\end{tabular}

Para la obtención de los datos experimentales se encendió el motor sin carga y se dejó en ralentí a las RPM mínimas hasta que alcance la temperatura de operación (aproximadamente $60^{\circ} \mathrm{C}$ en la culata). $\mathrm{Al}$ alcanzar la temperatura de operación se acelera el motor hasta 2800 RPM. Seguidamente se procede a encender el sistema hidráulico para aplicar el caudal estipulado del sistema que es 15 $\mathrm{Lt} / \mathrm{min}$, luego de que el sistema alcanza la estabilidad de temperatura la cual es de $72.9^{\circ} \mathrm{C}$, se procede a encender el ventilador del radiador para observar el comportamiento transitorio en la temperatura del sistema de enfriamiento dando como resultado una temperatura en régimen estable de $67.4{ }^{\circ} \mathrm{C}$ tal y como se muestra en la Fig. 9 , estos datos se obtuvieron con un datalogger del fabricante EXTECH instruments modelo SDL200 con una precisión de $\pm\left(0.4 \%+1{ }^{\circ} \mathrm{C}\right)$ para termocuplas tipo $\mathrm{k}$.

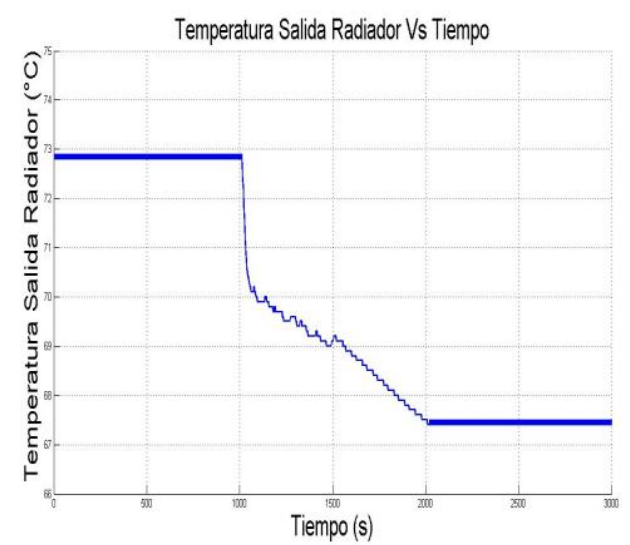

Fig. 9 Comportamiento de la temperatura de salida del radiador. Fuente autores.

Una vez obtenida la respuesta anterior se sobreponen los dos comportamientos de la Fig. 8 (simulado) y Fig. 9 (experimental) en la Fig. 10, para así realizar la respectiva validación del modelo.

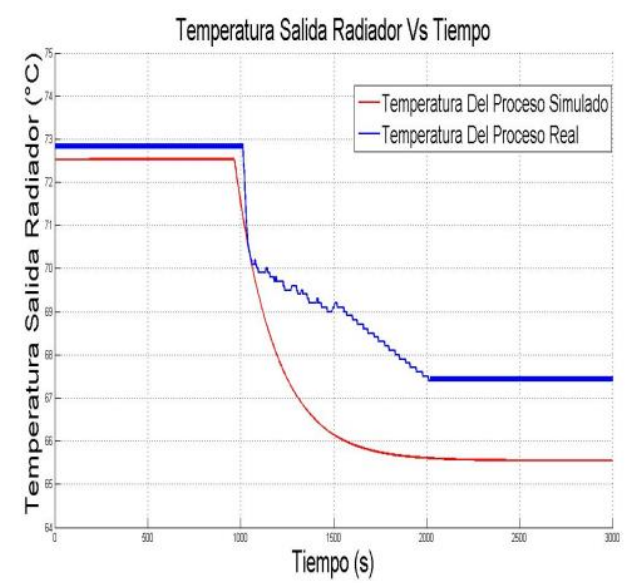

Fig. 10 Comportamiento del sistema con la temperatura obtenida del proceso real y la simulación del proceso.

Como se observó en la Fig. 10 el comportamiento simulado sigue la misma tendencia con respecto al proceso real, en la comparación de los dos procesos real y simulado se sobreponen las gráficas obteniendo el valor del error absoluto siguiendo la Ecuación 10 el cual representa la desviación del proceso simulado con respecto al proceso real, donde $T_{S}$ representa la temperatura simulada y $T_{R}$ representa la temperatura real, dando un valor de error de $4.6 \%$

$$
\varepsilon=\frac{\left|T_{s}-T_{R}\right|}{T_{R}} \cdot 100
$$




\section{CONCLUSIONES}

Cuando la temperatura del fluido de trabajo del dinamómetro alcanza $\operatorname{los} 72.5^{\circ} \mathrm{C}$ se conectó el ventilador del radiador logrando una remoción de calor de $6.54{ }^{\circ} \mathrm{C}$ en la temperatura de salida del radiador.

Se comprobó en el sistema de enfriamiento que al tener en cuenta el volumen del agua del reservatorio, las tuberías, el radiador y considerando que el flujo del aire que circula por el radiador fue calculado por el área de la circunferencia del ventilador se evidencio que el sistema arrojo buenos resultados ya que el comportamiento simulado tuvo la misma tendencia que el proceso real.

En la simulación se permite observar el comportamiento del sistema de enfriamiento puesto que al aumentar el caudal hay una disminución en el calor absorbido por el radiador y también se observa en la simulación que la velocidad del ventilador es proporcional al calor absorbido por el aire ya que si este aumenta también aumentara la remoción del calor absorbido por el aire.

En la comparación del proceso simulado con el proceso real se obtuvo un error absoluto de $4.6 \%$ permitiendo la validación del modelo matemático para representar el sistema de enfriamiento del dinamómetro hidráulico.

\section{REFERENCIAS}

Ariza F., C. y Vanegas R., L. (2013). Diseño de un Dinamómetro Hidráulico para Motores de Combustion Interna con una Potencia Menor a 65 HP, UPB, Bucaramanga.

Canizales O., J. y Zapata M., J. (2012). Diseño y Modelado de un Dinamometro Hidraulico para Pruebas de Motores, UTP, Pereira.

Çengel, Y. y Boles, M. (2012). Termodinámica, Mc Graw Hill, Ciudad de Mexico.

Estupiñan C., W. y Franco R., F. (2014). Determinación del Desempeño Mecánico, Energético y Ambiental de un Motor Diesel Mono Cilindrico, para Efectuar su Funcionamiento con Aire Enriquecido de sus Productos de la Hidrolisis del Agua, UFPS, Cúcuta.

Hodson, P. (1991). Theoretical Model and Dynamic Simulation of Variable Fill Hydraulic Dynamometers, New Zealand.

KCP López, BM Delgado..., (2017), Efecto De La Dispersión Cromática En Un Sistema Híbrido Revista Colombiana de
Tecnologías de Avanzada ISSN: 16927257.

Mejia C., L., Monroy J., M., Diaz A., A., Henao C., E. y Hoyos M., M. (2003). Modelado y Simulacion del Control de Velocidad de un Motor Hidráulico,UTP, Pereira.

Narayan R., N. (1968). The Basic Theory Hydraulic Dynamometers nd Retardes, India.

RÁ López, MG Angarita. (2017), Convertidores Fotovoltaicos En Módulos Integrados Basados En Inversores De Fuente-SemiCuasi-Z: Un Nuevo Esquema De ... Revista Colombiana de Tecnologías de Avanzada ISSN: 1692-7257

Romero P., C. y Carranza S., Y. (2007). Elementos de Diseño de Sistemas de Enfriamiento de Motores de Combustion Interna, UTP, Pereira.

Verdesoto C., G. (2006). Diseño y Construcción de un Radiador de Automovil Para el Chevrolet Corsa 1300 y Elaboracion de un Sofware para el Diseño, Latacunga.

\section{ANEXOS}

$c_{\text {paire }} \quad$ Calor Específico a presión del Aire

$T_{\text {eair }} \quad$ Temperatura de Entrada del aire

$T_{\text {sair }} \quad$ Temperatura de Salida del aire

$T_{\text {entragu }} \quad$ Temperatura de Entrada del Agua

$\rho_{\text {aire }} \quad$ Densidad del Aire

$\rho_{\text {agua }} \quad$ Densidad del Agua

$V_{\text {radiador }}$ Volumen de Agua del Radiador

$V_{\text {reservatorio }}$ Volumen de Agua del Reservatorio

$V_{\text {tuberias }}$ Volumen de Agua por las Tuberías

$c_{\text {pagua }} \quad$ Calor Específico a Presión del Agua

$q \quad$ Caudal

$C_{\text {vagu }} \quad$ Calor especifico a Volumen Constante

$V_{\text {aire }} \quad$ Velocidad del Aire

$m_{\text {aire }} \quad$ Flujo másico de aire

aire Tlujo másico de aire

$m_{\text {agua }} \quad$ Flujo másico de agua

$T_{S} \quad$ Temperatura simulada

$T_{R} \quad$ Temperatura real 